\title{
Effect of dipolar interactions on the phase behavior of the Gay-Berne liquid crystal model
}

\author{
Mohammed Houssa and Luis F. Rull \\ Departamento de Física Atómica, Molecular y Nuclear, Area de Física Teórica, Universidad de Sevilla, \\ Aptdo 1065, Sevilla 41080, Spain \\ Simon C. McGrother \\ School of Chemical Engineering, North Carolina State University, Raleigh, North Carolina 27695-7905
}

(Received 29 May 1998; accepted 21 August 1998)

\begin{abstract}
A computer simulation study of the phase behavior of the dipolar Gay-Berne liquid crystal model is presented. The phase transitions are determined with isothermal-isobaric (NPT) Monte Carlo simulations, utilizing the reaction field method. The electrostatic forces are found to have a considerable effect on the nature of the observed phases, but the density at which the isotropic fluid becomes unstable with respect to partially ordered phases is seen to be remarkably insensitive to the strength of the dipole. We pay particular attention to the structure of the mesophases, combining information from several singlet and pair distribution functions to build up an accurate picture of the molecular arrangement of the systems. (C) 1998 American Institute of Physics.

[S0021-9606(98)51544-9]
\end{abstract}

\section{INTRODUCTION}

It is becoming increasingly evident that the liquid crystalline phase behavior of complex molecules is dictated, principally, by the nonsphericity of the inflexible regions of the constituent molecules. Since the 1940's, it has been recognized that the simplest liquid crystal (the nematic) could result from excluded volume effects only. In more recent years, other, more complex, liquid crystalline phases have been reported for model molecules interacting only via anisotropic repulsive potentials. Both smectic ${ }^{1}$ and columnar ${ }^{2}$ phases have been observed for these simple models. Even the spontaneous polarity of the tilted chiral smectic-C* phase has been rationalized purely in terms of packing effects of the repulsive cores. ${ }^{3}$

Of course, it is only in these idealized theoretical models that the attractive and repulsive elements of the intermolecular interaction can be considered in isolation. In any real system, both forces will be present. There are, however, colloidal systems (e.g., tobacco mosaic virus), in which the nonspherical molecules have a negligible attraction for each other and the phase transitions are thought to be almost entirely driven by excluded volume effects. True to theoretical prediction, such systems do indeed exhibit a number of mesophases at appropriate concentrations and temperatures. But these systems are exceptional; in all thermotropic mesogens, attractive forces are not only present, but frequently comprise complicated components such as multipolar forces and $\pi$-electron interactions, in addition to the usual London dispersion effects. It is important, therefore, that the role of the attractive forces in stabilizing the mesophases is investigated.

Frenkel and co-workers have shown that hard prolate and oblate ellipsoids of revolution ${ }^{4}$ and hard spherocylinders ${ }^{5}$ have a rich phase diagram with nematic, and in some cases smectic and columnar phases, depending on the molecular size and shape. These findings reinforce the ideas of Onsager, ${ }^{6,7}$ that the anisotropic shape of the molecules is the principal driving force behind mesophase formation. The attractive forces (van der Waals, multipolar, dispersion, etc.) are of secondary importance. Despite their lesser role, an investigation of the influence of such attractive forces is necessary if a full understanding of liquid crystalline phase behavior is to be achieved. Probably the simplest idealized liquid crystal (LC) model which incorporates both repulsive and attractive terms, and consequently the most widely studied, is the Gay-Berne (GB) potential. Depending upon the choice of parameters this model may be either oblate (disclike) or prolate (rodlike). The majority of real mesogens are rod shaped, thus we focus in this study on the prolate GayBerne model. Both the shape- and energetic anisotropy are adjustable. A minimum elongation is required before orientationally ordered fluid phases become thermodynamically stable. Similarly, there is a minimum energetic-anisotropy, below which no spatially inhomogeneous fluid phases are observed. These two minimum values are partially coupled.

Obviously there is a vast parameter space to explore; it is necessary, therefore, to limit our investigation to a single energetic anisotropy $\kappa^{\prime}=5\left(\kappa^{\prime}\right.$ is the ratio of the potential well depths of side-by-side and end-to-end configurations), a value which yields a rich phase diagram for elongations of interest.

Studies of the Gay-Berne potential with $\kappa=3(\kappa$ is the molecular elongation, and is defined as the ratio of the two principal axes of the ellipsoidal core) and $\kappa^{\prime}=5$ have proven that the isotropic (I), nematic $(\mathrm{N})$, smectic-B $(\mathrm{SmB})$, and crystalline phases can be formed along different isotherms. ${ }^{8,9}$ Upon increasing the elongation of the GB particle (i.e., $\kappa$ $>3)$, the smectic-A (SmA) phase appears between the nematic and smectic-B phases, for certain temperatures. For hard ellipsoids with an elongation $a / b=3$ (with $a$ and $b$ the 
lengths of the two principal molecular axes), the isotropic and crystalline phases are separated only by a nematic region. Thus we can confidently state that the existence of the smectic phases is directly related to the attractive forces in the GB model (the softness of the repulsion is unlikely to cause a qualitative difference in phase behavior).

Studies of dipolar liquid crystal models have also been reported in recent years. Weis, Levesque, and Zarragoichoechea, in a series of papers, ${ }^{10-13}$ studied how the presence of point dipoles influenced the structure of the LC phases of a variety of hard-core models. Selected state points, representative of various mesophases, were simulated with Monte Carlo, employing the computationally expensive Ewald summation method, principally in the canonical ensemble. Two major conclusions were drawn: central dipoles promote layered structures and terminal dipoles have little effect on the structure of the phase. Little could be concluded as to the stability of the various phases, since only a very limited number of state points were investigated. McGrother et al. constructed full isotherms for several spherocylinder plus dipole models. For central dipoles, ${ }^{14}$ short-ranged order in the isotropic phase made the I-LC phase transition difficult to pinpoint; indeed, for sufficiently strong dipoles, compression led only to metastable glassy phases and no true LC phases were observed. A much greater degree of hysteresis is noted at the I-N transition than in the hard spherocylinder system. The presence of the central dipole (both longitudinal ${ }^{14}$ and transverse ${ }^{15}$ ) unambiguously stabilizes the smectic-A phase. This layered phase is seen to be stable at pressures and densities corresponding to less ordered phases in the nonpolar system. When the dipole resides in the terminal position, ${ }^{16}$ different behavior is noted. Again hysteresis is seen at the I-N transition, but now the smectic-A phase is destabilized, and the $\mathrm{N}-\mathrm{SmA}$ transition is postponed to higher density and pressure as a result of the electrostatic interaction. In all cases no ferroelectric phases were noted.

For the Gay-Berne model, some numerical results for dipolar systems have been offered. Principally Satoh et al. ${ }^{17,18}$ have used the reaction field method to create several isochores for such systems. For GB molecules $(\kappa=3$ and $\kappa^{\prime}=5$ ) with central longitudinal dipoles, ${ }^{17}$ the I-N transition is again seen to be insensitive to the dipole strength. On the other hand, the $\mathrm{N}-\mathrm{SmA}$ transition temperature is seen to increase with the dipole moment, i.e., the smectic-A phase is stabilized. The temperature at which crystallization occurs does not vary much with the strength of the multipole. In terms of structure, the layering in the smectic-A phase is observed to be much sharper in the dipolar system, at low temperatures; near the $\mathrm{N}-\mathrm{SmA}$ transition, the effect is less pronounced. When the longitudinal dipole is placed in the terminal position, ${ }^{18}$ the I-N transition temperature $T_{\mathrm{IN}}$ increases with increasing dipole moment $\mu^{*}=\left(\mu^{2} / \varepsilon_{0} \sigma_{0}^{3}\right)^{1 / 2}$ where $\sigma_{0}$ is the contact distance, and $\varepsilon_{0}$ is the energy (ignoring the dipole) of a pair of GB molecules in the side-byside arrangement. $T_{\mathrm{NS}}$ exhibits similar $\mu$ dependence. The structure of the resultant smectic phase is seen to be bilayered, with a notable degree of interdigitation.

Interesting phases were found by Berardi et al. ${ }^{19}$ when the longitudinal dipole is in either the central or terminal position. This study focused primarily on the smectic phases, and as such large system sizes were employed $(N=1000$ and 8000). Three temperatures were selected at a fixed density $\rho^{*}=\rho \sigma_{0}^{3}=0.3$, corresponding, in the absence of dipolar interactions, to isotropic, nematic, and smectic-A phases, respectively. The addition of point dipoles with $\mu^{*}=2$ is seen to have no influence on the overall nature of the observed phase, at least at the three chosen temperatures. In both LC phases, the presence of the dipole in either position is seen to increase the orientational order slightly. For central dipoles, the smectic has the usual monolayer structure, but a characteristic split of the second peak in the radial pair distribution function leads Berardi et al. to describe this phase as smectic-B. Shifting the dipole toward one end of the molecule eliminates this hexagonal order within the plane, but several new features are noted. The projection of the pair distribution function shows a splitting in the first peak, which is indicative of a bilayer structure. The first peak is at a distance which suggests significant interdigitation. Particles in each layer are locally ferroelectric (i.e., the dipoles all point in the same direction), and the direction of polarization alternates between successive layers. These findings suggest that the phase is a bilayered antiferroelectric smectic phase. However, simulation of huge systems $(N=8000)$, and visualization of the phase, shows that the situation is somewhat more complex. In each of the smectic layers, there is a noticeable dislocation, at which the center of the layer shifts by around a molecular length (due to the toroidal boundaries, there is second dislocation in each layer, which cancels the effect of the first). Even starting in a perfect smectic-A phase, the system was seen to relax into the eloquently named modulated antiferroelectric bilayer stripe domains.

Gwóźdź et al. ${ }^{20}$ used molecular dynamics (MD) to study the influence of transverse central dipoles on the phase diagram. These authors take a slightly different approach: rather than cooling along an isochore, they compress along an isotherm, for only one value of the dipole moment $\left(\mu^{*}\right.$ $=0.5$ ). They find that the I-SmA transition occurs at the same density with or without the dipolar forces present. The only distinctions between the polar and nonpolar case are the higher degree of pretransitional order in the dipolar system, and better defined positional order in the smectic phase. The authors report smectic-C order in the dipolar case, but the tilt angle is small and the tilt disappears as the density is increased still further.

Most recently Houssa et al. ${ }^{9}$ studied a GB model decorated with a central longitudinal dipole. These authors report the complete suppression of the nematic phase for sufficiently strong dipoles. The phase sequence for the nonpolar system is $\mathrm{I}-\mathrm{N}-\mathrm{SmB}$, but with the inclusion of a dipole of reduced moment $\mu^{*}=2.5$, the isotropic phase spontaneously orders directly to the smectic-B phase upon compression.

Thus from earlier studies of dipolar LC models we can conclude that the dipole causes only a mild perturbation to the initial I-LC transition, which appears to be almost entirely dependent upon the short-range repulsive forces acting between anisotropic molecules. Depending on the position and orientation of the dipole in the molecular frame, the nematic or smectic phases can be promoted at the expense of 
the other. The most evident feature of all previous studies is that the fine detail of the smectic structure is sensitive to the dipole. Interdigitation, bilayers, striped domains, and even tilted phases are reported for various systems.

In the next section we describe the potential model, and in Sec. III we give details of the simulation methodologies that have been employed. The results of the simulation studies are presented and discussed in Sec. IV, and we draw our conclusions in the final section.

\section{POTENTIAL MODEL}

In our simulations, the interaction energy of two molecules $i$ and $j$ is given by

$$
U_{i j}=U_{\mathrm{GB}}\left(\hat{\mathbf{r}}_{i j}, \hat{\mathbf{u}}_{i}, \hat{\mathbf{u}}_{j}\right)+U_{\mu \mu}\left(\hat{\mathbf{r}}_{i j}, \hat{\mathbf{u}}_{i}, \hat{\mathbf{u}}_{j}\right),
$$

where $U_{\mathrm{GB}}\left(\hat{\mathbf{r}}_{i j}, \hat{\mathbf{u}}_{i}, \hat{\mathbf{u}}_{j}\right)$ is the GB potential ${ }^{21}$

$$
\begin{aligned}
U_{\mathrm{GB}}\left(\hat{\mathbf{r}}_{i j}, \hat{\mathbf{u}}_{i}, \hat{\mathbf{u}}_{j}\right)= & 4 \varepsilon\left(\hat{\mathbf{r}}_{i j}, \hat{\mathbf{u}}_{i}, \hat{\mathbf{u}}_{j}\right) \\
& \times\left[\left(\frac{\sigma_{0}}{r_{i j}-\sigma\left(\hat{\mathbf{r}}_{i j}, \hat{\mathbf{u}}_{i}, \hat{\mathbf{u}}_{j}\right)+\sigma_{0}}\right)^{12}\right. \\
& \left.-\left(\frac{\sigma_{0}}{r_{i j}-\sigma\left(\hat{\mathbf{r}}_{i j}, \hat{\mathbf{u}}_{i}, \hat{\mathbf{u}}_{j}\right)+\sigma_{0}}\right)^{6}\right],
\end{aligned}
$$

where $\hat{\mathbf{u}}_{i}$ is the axial vector of molecule $i$ and $r_{i j}$ is the distance between the centers of mass of molecules $i$ and $j$, $\hat{\mathbf{r}}_{i j}=\mathbf{r}_{i j} / r_{i j}$ is a unit vector along the intermolecular vector $\mathbf{r}_{i j}=\left|\mathbf{r}_{i}-\mathbf{r}_{j}\right|$, where $\mathbf{r}_{i}$ and $\mathbf{r}_{j}$ are the positions of the centers of mass of molecules $\mathrm{i}$ and $\mathrm{j}$, respectively. Here $\sigma\left(\hat{\mathbf{r}}_{i j}, \hat{\mathbf{u}}_{i}, \hat{\mathbf{u}}_{j}\right)$ and $\varepsilon\left(\hat{\mathbf{r}}_{i j}, \hat{\mathbf{u}}_{i}, \hat{\mathbf{u}}_{j}\right)$ are the range and strength parameters, respectively (see Ref. 21 for explicit expressions), $\sigma$ and $\varepsilon$ depend on the anisotropy parameters $\kappa$ (molecular elongation) and $\kappa^{\prime}$ (energetic anisotropy). $\sigma_{0}$ and $\varepsilon_{0}$ (the range and energy values in the side-by-side arrangement) are used to define the pressure and temperature scales in our simulations. Thus $P^{*}=P \sigma_{0}^{3} / \varepsilon_{0}$ and $T^{*}=k T / \varepsilon_{0}$. The dipole-dipole interaction is given by

$$
U_{d d}\left(\hat{\mathbf{r}}_{i j}, \hat{\mathbf{u}}_{i}, \hat{\mathbf{u}}_{j}\right)=\frac{\left[\boldsymbol{\mu}_{i} \boldsymbol{\mu}_{j}-3\left(\boldsymbol{\mu}_{i} \cdot \hat{\mathbf{r}}_{i j}\right)\left(\boldsymbol{\mu}_{j} \cdot \hat{\mathbf{r}}_{i j}\right)\right]}{r_{i j}^{3}},
$$

where $\boldsymbol{\mu}_{i}=\mu \hat{\mathbf{u}}_{i}$ and $\boldsymbol{\mu}_{j}=\mu \hat{\mathbf{u}}_{j}$ denote the dipole moments of molecules $i$ and $j$, respectively. The dipoles are longitudinal, i.e., parallel to the unit vectors which represent the principal molecular axes $\hat{\mathbf{u}}_{i}$ or $\hat{\mathbf{u}}_{j}$.

While the GB potential $U_{\mathrm{GB}}\left(\hat{\mathbf{r}}_{i j}, \hat{\mathbf{u}}_{i}, \hat{\mathbf{u}}_{j}\right)$ can be truncated and shifted at some distance, $r_{c}$, less than $L / 2, L$ being the length of the simulation box, the same is not true for the dipolar interaction. The long-ranged nature of the dipolar potential is taken into account with the reaction field method. This scheme ${ }^{22}$ assumes that particles beyond a cutoff distance, $r_{c}$, act as a dielectric continuum of dielectric constant $\varepsilon_{\mathrm{RF}} \cdot{ }^{23}$ The interaction energy for a pair of dipoles can then be written

$$
U_{\mu \mu}\left(\hat{\mathbf{r}}_{i j}, \hat{\mathbf{u}}_{i}, \hat{\mathbf{u}}_{j}\right)=U_{d d}\left(\hat{\mathbf{r}}_{i j}, \hat{\mathbf{u}}_{i}, \hat{\mathbf{u}}_{j}\right)-\frac{2\left(\varepsilon_{\mathrm{RF}}-1\right)}{2 \varepsilon_{\mathrm{RF}}+1} \frac{\boldsymbol{\mu}_{i} \cdot \boldsymbol{\mu}_{j}}{r_{c}^{3}},
$$

for $r_{i j}<r_{c}$ and $U_{\mu \mu}\left(\hat{\mathbf{r}}_{i j}, \hat{\mathbf{u}}_{i}, \hat{\mathbf{u}}_{j}\right)=0$ for $r_{i j}>r_{c}$.

\section{SIMULATION DETAILS}

The simulations were performed using Monte Carlo in the isothermal-isobaric ensemble (i.e., constant number of molecules $N$, pressure $P$, and temperature $T$ ) with the reaction field method. Each Monte Carlo cycle consists of $N$ trial displacements and reorientations of the GB molecules and approximately one trial volume change. The maximum displacement, rotation, and volume change are altered to ensure that around $40 \%$ of each type of move is accepted; this value should lead to the most efficient sampling of phase space. The reaction field is a simple but accurate method for accounting, in an average way, for the long range of the dipolar interaction. The technique is much faster than the Ewald summation method, but yields results which are essentially indistinguishable. For comparison of these two methods see Refs. 9, 24, and 25.

In order to analyze the orientational order and the possible polarization of the mesophases, we have calculated two order parameters $S$ and $P_{1}$; the former (the nematic order parameter) is obtained as the largest positive eigenvalue of the $Q$ tensor, ${ }^{26}$ the elements of which are defined as

$$
Q_{\alpha \beta}=\frac{1}{N} \sum_{i=1}^{N} \frac{1}{2}\left(3 u_{\alpha}^{i} u_{\beta}^{i}-\delta_{\alpha \beta}\right)
$$

( $u_{k}^{i}$ is the $k$ th component of the axial orientation $\hat{\mathbf{u}}_{i}$ of the $i$ th molecule), with the nematic director $\mathbf{n}$ being the corresponding eigenvector. The polarity $P_{1}$ is obtained as

$$
P_{1}=\frac{1}{N}\left|\sum_{i=1}^{N} \hat{\mathbf{u}}_{i} \cdot \mathbf{n}\right| \text {. }
$$

The nematic order parameter, $S$, is zero in the isotropic phase, and has a value of one in a perfectly aligned phase; it provides no information as to positional order within the system. In real systems, $S$ attains values around 0.4 at the I-N transition, whereas in simulation, higher values are frequently encountered due to finite size effects. The polarity $P_{1}$ is also zero in the isotropic phase, can only attain its maximum value (one) in a perfectly aligned state, and similarly gives no information on the positional order within the system. The functions are distinct because $P_{1}$ lacks "headtail" symmetry, and is only nonzero when more of the dipoles "point'" in one direction than another. As such, $P_{1}$ measures the spontaneous polarization of the phase.

We calculate various pair distribution functions, e.g., the first-rank orientational correlation function defined by

$$
g_{1}(r)=\left\langle P_{1}\left(\hat{\mathbf{u}}_{1}, \hat{\mathbf{u}}_{2}\right)\right\rangle=\left\langle\cos \theta_{12}\right\rangle,
$$

and the second-rank orientational correlation function,

$$
g_{2}(r)=\left\langle P_{2}\left(\hat{\mathbf{u}}_{1}, \hat{\mathbf{u}}_{2}\right)\right\rangle=\left\langle\frac{3}{2} \cos ^{2} \theta_{12}-\frac{1}{2}\right\rangle,
$$

where $\theta_{12}$ is the angle between the principal molecular axes of molecules 1 and 2 . In addition, we also determine the orientationally averaged pair correlation functions for two molecules whose centers lie on a line parallel, $g_{\|}\left(r_{\|}\right)$, or on a line perpendicular, $g_{\perp}\left(r_{\perp}\right)$, to the director. Here the distances $r_{\|}$and $r_{\perp}$ are measured parallel and perpendicular to the director, respectively. 

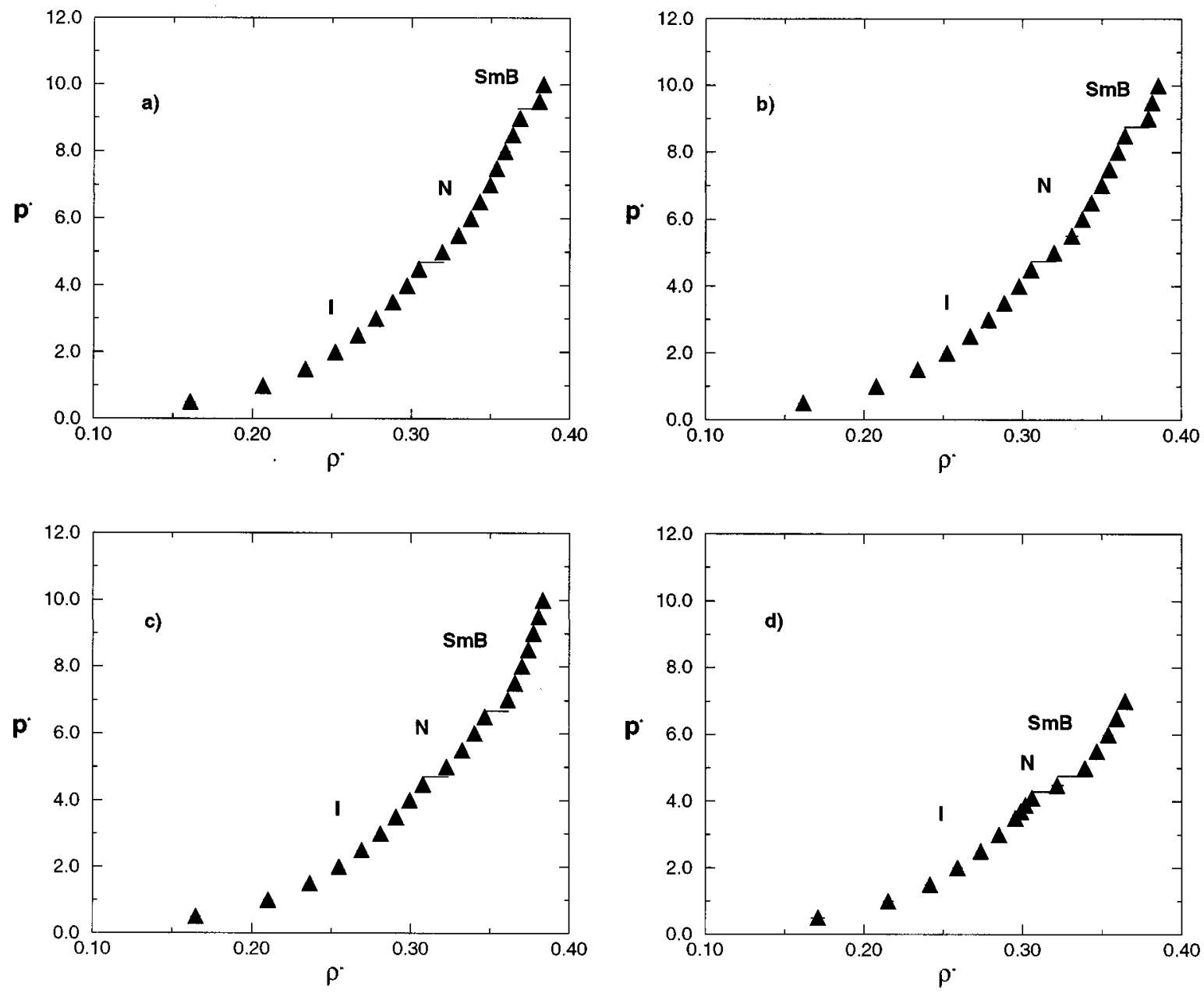

FIG. 1. Phase diagram at $T^{*}=1.25$, of a system of 256 Gay-Berne particles $\left(\kappa=3, \kappa^{\prime}=5\right)$ with embedded central, longitudinal point dipoles. The reduced pressure $P^{*}=P \sigma_{0}^{3} / \varepsilon_{0}$ is plotted as a function of the reduced number density $\rho^{*}=\rho \sigma_{0}^{3}$. Each plot depicts a different reduced dipole moment $\mu^{*}$ $=\left(\mu^{2} / \varepsilon_{0} \sigma_{0}^{3}\right)^{1 / 2}$ (a) $\mu^{*}=0.5$, (b) $\mu^{*}=1.0$, (c) $\mu^{*}=1.5$, and (d) $\mu^{*}=2.0$. Error bars denote one standard error in the density. Horizontal lines denote the position of the phase transitions.

These functions alone cannot unequivocally determine the nature of certain smectic phases. To verify the structure of these liquid crystals, we calculate the bond-orientational order within the smectic layers. This function is defined as

$$
B=\frac{1}{6 N}\left\langle\sum_{j=1}^{6} \exp \left(6 i \phi_{i j}\right)\right\rangle,
$$

where $\phi_{i j}$ is the angle between the bond linking particles $i$ and $j$ and a fixed reference axis. The sum is over only nearest-neighbor bonds. We define nearest-neighbor bonds to be those within $r_{b} \approx 1.2 \sigma_{0}$. This order parameter takes values close to zero when no in-plane bond order exists and close to one in the presence of perfect hexatic in-plane bond order. Principally this parameter is used to distinguish the smectic-A phase from the more ordered smectic-B structure.

For GB molecules with $\kappa=3$, we have simulated $N$ $=256$ molecules with longitudinal point dipoles located at the center of the molecules, with dipole moment $\mu^{*}$. In order to minimize the effect of the simulation cell on the observed phases, a cubic simulation box was employed with periodic boundary conditions. For more elongated particles $(\kappa=4)$ we are forced to examine larger systems $(N=500)$, in a cuboidal cell. Dipole moments ranging from $\mu^{*}=0.5$ to 2.5 (in steps of 0.5 ) were considered. For the smaller system, at each value of $\mu^{*}$, simulations were initiated from a facecentered-cubic (fcc) lattice with five layers perpendicular to the $z$-axis, which is expanded to a low density $\rho^{*}=\rho \sigma_{0}^{3}$ $=0.08\left(P^{*}=0.5\right)$ and quickly loses positional and orientational order. The system was then slowly compressed in reduced pressure steps of 0.5 or less. For the larger system, states in the isotropic, nematic, and smectic-A phase were generated with nonpolar GB systems. The dipole is introduced and the system allowed to equilibrate once more. Typically, between 3 and $5 \times 10^{5}$ cycles were performed for each state point, increasing to $10^{6}$ cycles in the vicinity of phase transitions.

By modern standards these system sizes are moderate; however, we performed several simulations with larger system sizes and noted no systematic difference in the results. Furthermore, simulating small systems allows us to explore phase space much more thoroughly. At the liquid crystalline phase transitions, large molecular reorganizations must occur and lengthy runs are crucial. We are confident that system sizes are sufficient to determine the nature of the phases and 

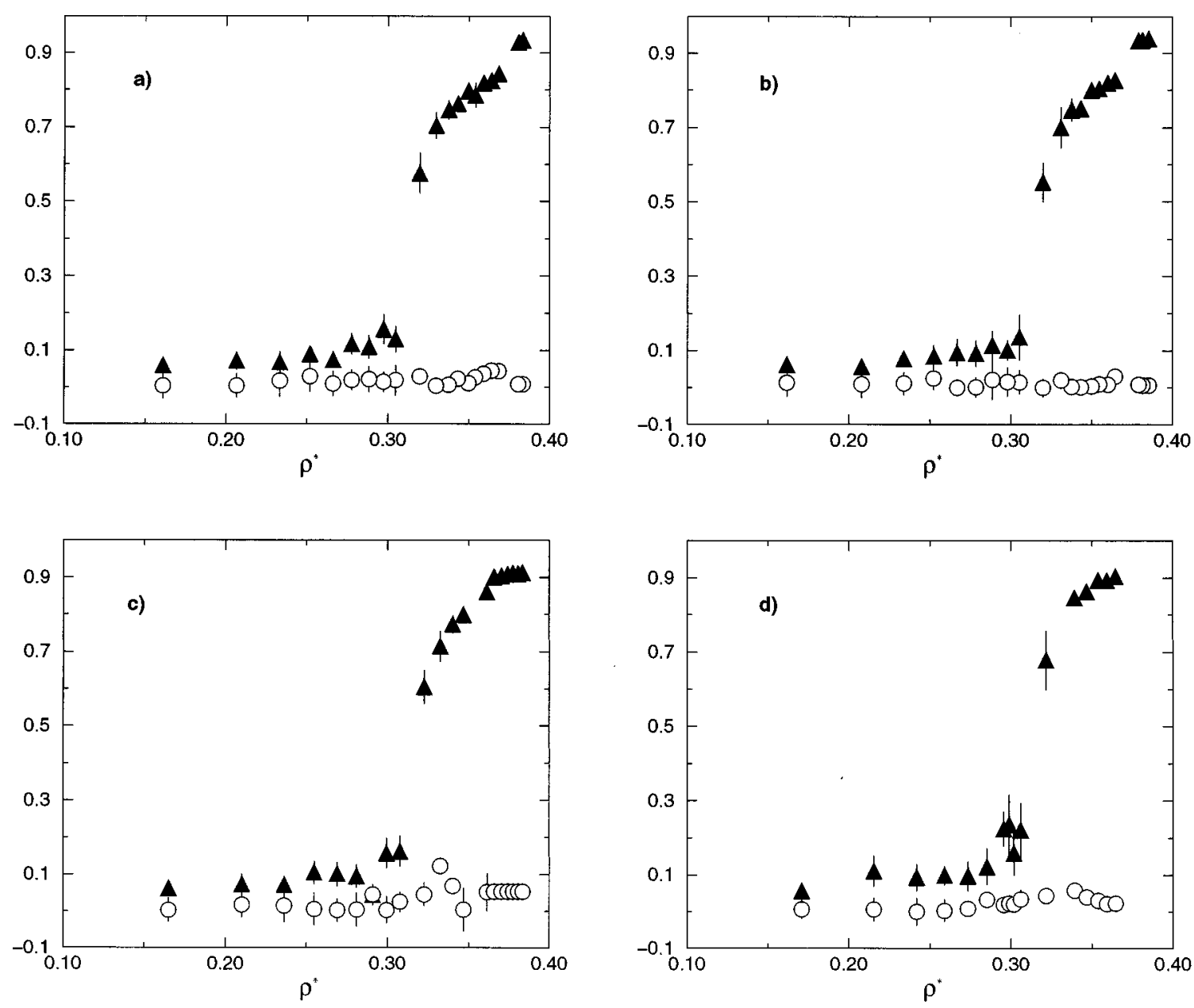

FIG. 2. Variation of the nematic order parameter $S$ (triangles) and polarity $P_{1}$ (circles), as a function of density for the dipolar Gay-Berne fluid. The four figures correspond to the same dipole moments as Fig. 1. Error bars denote one standard error in the order parameters.

accurately predict the thermodynamic properties of the system. This belief is given credence by the excellent agreement noted between our simulations and those of Brown et al. ${ }^{28}$

In this paper we present the results of a series of NPT Monte Carlo (MC) simulations on a system of molecules interacting via the Gay-Berne dipolar potential with $\kappa=3$ or 4 and $\kappa^{\prime}=5$.

\section{SIMULATIONS RESULTS}
A. $\kappa=3$
1. $T^{*}=1.25$

At this temperature we have constructed isotherms for various dipolar strengths. For each value of $\mu^{*}$, the lowest pressure simulated corresponds to an isotropic fluid, and increasing the pressure eventually leads to at least one phase transition, indicated by a small discontinuity in the density and a marked increase in orientational order.

Focusing our attention on weaker dipole moments $\mu^{*} \leqslant 2.0$, the numerical phase diagrams are presented in Fig. 1 , with the pressure dependence of the orientational order parameter $S$ and polarity $P_{1}$ given in Fig. 2. From these figures we can perceive two distinct transitions. The first transition is from $S$ values around zero (isotropic phase) up to nonzero values typical of the nematic phase. That the re- sultant phase is nematic, can be confirmed by inspection of the projections of the pair distribution functions parallel $g_{\|}\left(r_{\|}\right)$and perpendicular $g_{\perp}\left(r_{\perp}\right)$ to the director (Figs. 3 and 4). These figures show that the structure is liquidlike and that there is no significant layering at the lower of the two indicated pressures. The second transition is from moderate values of $S$, up to saturation $S \approx 0.94$. This high density phase is best characterized by $g_{\|}\left(r_{\|}\right)$(Fig. 3): the sinusoidal variation of this function indicates that there is one-dimensional layering of particles, which is the trademark of the smectic liquid crystals. The wavelength of this periodic function is indicative of the layer spacing of the smectic strata and the amplitude is related to how well defined the layers are. It is clear that the strength of the dipole does not significantly affect either the wavelength or the amplitude of this function at the nematic-smectic transition. This result is quite surprising; previous simulation studies have indicated the significant impact of the dipole upon the details of the structure, particularly of the ordered phases. The striking similarity of these figures shows that the structure of the smectic liquid crystal is dependent principally on the GB interactions. The layer spacing (see Fig. 3) is slightly less than one molecular length, which is a common feature of such phases when the constituent molecules have an ellipsoidal core: the tapering of the molecule permits a degree of interdigitation and this 

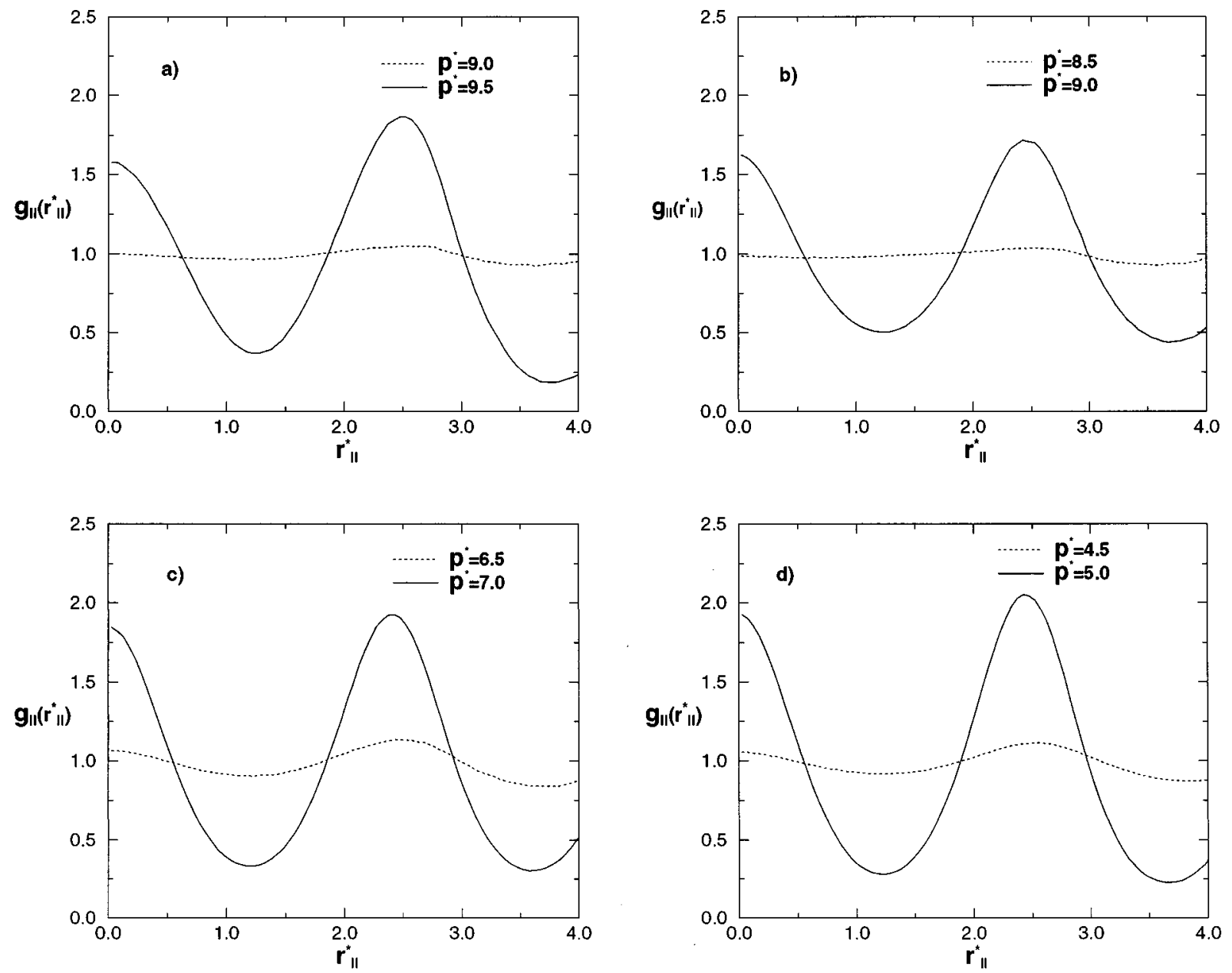

FIG. 3. The projection of the radial pair distribution function parallel to the director $g_{\|}\left(r_{\|}\right)$for states on either side of the nematic-smectic transition for the same systems as Fig. 1.

structure has the most efficient packing of particles. From Fig. 4 the N-Sm transition is also accompanied by an increase in the perpendicular order. Significantly, a peak develops at less than one diameter, corresponding to nearest neighbors in the next smectic stratum. The long-ranged nature of this function in the smectic phase indicates that the phase is more highly structured than a simple smectic-A phase. In Fig. 5 we show a snapshot of the $\mu^{*}=1.5$ system at a reduced pressure $P^{*}=9.0$, in this smectic phase. The smectic layers are clear from Fig. 5(a), and the positional order within those layers is evident from Fig. 5(b). The $\mathrm{N}-\mathrm{Sm}$ transition is accompanied by a distinct increase in the bond-orientational order. Thus we conclude that the phase is smectic-B. Interestingly, as the strength of the dipole is increased, the number of peaks perpendicular to the director is seen to diminish. The orientationally averaged pair distribution function, Fig. 6, exhibits the expected oscillatory behavior and high value at long distances, of the layered smectic phases. From Fig. 6 we see that there is significant shortrange orientational order in the isotropic phase near to the $\mathrm{I}-\mathrm{N}$ transition. This order is seen to increase slightly with increasing dipole moment. After the I-N transition these orientational correlations no longer fade to zero within the simulation cell. The orientational correlation at contact is an increasing function of $\mu$. In Fig. 7 we display the first-rank orientational correlation function for states in the isotropic nematic and smectic-B phases for these four dipole moments. Negative values indicate that particles are antiparallel. The figures are qualitatively equivalent; each shows that: nearest neighbors are antiparallel; next nearest neighbors are oriented parallel to the selected particle; the minima and maxima increase in magnitude as one moves from isotropic to nematic to smectic phases; the order dies out within the simulation cell, indicating no globally polar phases.

Both of the transitions ( $\mathrm{I}-\mathrm{N}$ and $\mathrm{N}-\mathrm{SmB}$ ) are seen to be weakly first order. Neither phase change affects the polarity $P_{1}$, which remains essentially zero, over the entire pressure range. Overall, these systems have the phase sequence I-N$\mathrm{SmB}$ on compressing from low density at this temperature $\left(T^{*}=1.25\right)$. This is true for nonpolar GB particles and those with weak, central, longitudinal dipoles.

A natural question is why the saturation value of $S$ is less than one. A perfectly aligned state is never achieved in simulations as a result of several particles becoming trapped at right angles to the director. These particles are particularly noticeable in the smectic phases, where they prefer to position themselves in the interlayer region. This fact is the source of the characteristic minimum in the orientational pair distribution function of simulated smectic liquid crystals. 

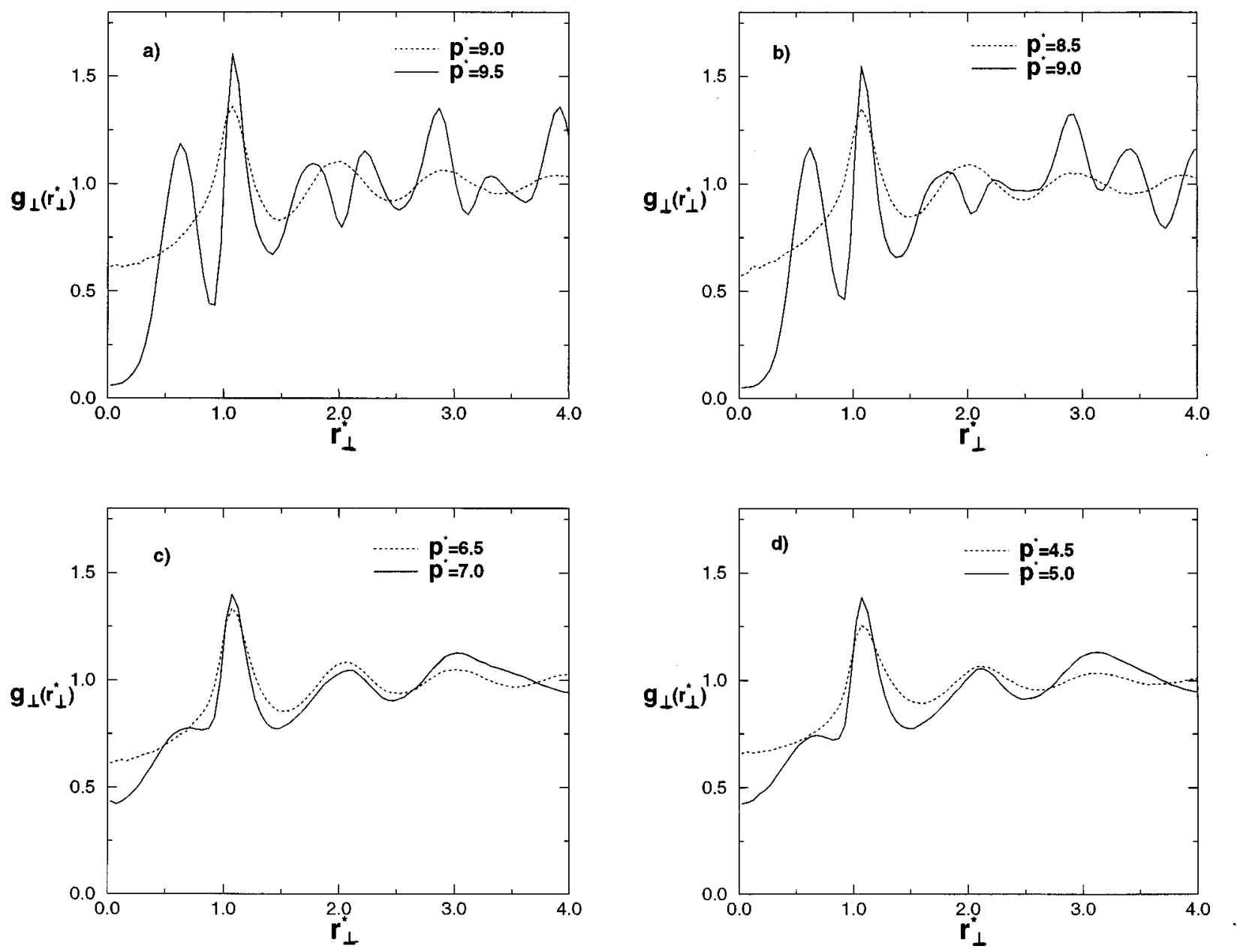

FIG. 4. The projection of the radial pair distribution function perpendicular to the director $g_{\perp}\left(r_{\perp}\right)$ for the same states as Fig. 3 .

The behavior of these molecules has been studied in detail for related systems. ${ }^{27}$

In Table I we report our estimates of the $\mathrm{I}-\mathrm{N}$ and $\mathrm{N}-$ $\mathrm{SmB}$ transition pressures and coexisting densities for the different dipolar strengths. From this table, it is clear that the introduction of the longitudinal dipole into the model has some impact on the I-N transition pressure, but the strength of the dipole has little effect upon the density of the isotropic phase at the transition. The density of the nematic phase that coexists with the isotropic fluid is also unchanging with variations in dipole moment. The $\mathrm{I}-\mathrm{N}$ transition pressure $\left(P_{\mathrm{IN}}\right)$ is seen to fall with increasing dipole moment. The consistency of the I-N transition densities $\rho_{\text {IN }}$, which are, to within experimental error, identical for each of the dipole strengths which lead to an I-N transition $\left(\mu^{*} \leqslant 2.0\right)$, is striking. At the layering transition $(\mathrm{N}-\mathrm{SmB})$, we now see that the densities at coexistence decrease as the strength of the multipolar interaction is increased. The transition pressure shows a very clear trend, moving to lower values as the dipole moment is increased. This is in accordance with expectation: longitudinal dipoles are well known to promote layered structures in both numerical and experimental studies, thus the stabilization of the smectic phase is to be expected. Increasing the dipole makes the smectic-B phase stable at lower pressures.

Configurations at $P^{*}=7.0$ were used as starting points for decreasing the pressure. A limited number of expansion runs have been performed to gauge the level of hysteresis at the transitions. For these weak dipoles $\left(\mu^{*} \leqslant 2.0\right)$, we see no evidence of hysteresis at the I-N transition; however, at the $\mathrm{N}-\mathrm{SmB}$ phase change, the transition pressure is very different depending on the direction from which the transition is approached. The same is true of the $\mathrm{N}-\mathrm{SmB}$ transition density, but since the phase diagram is very steep in this region (i.e., large changes in pressure correspond to only small changes in density), the effect is less obvious.

The natural consequence of the trends noted for the weaker dipoles in Table I is that the nematic phase will disappear at a triple point as the strength of the dipole moment is increased. This is due to the increasing stabilization of the smectic-B phase by the dipole, leading eventually to the layered phase preempting the nematic phase when the multipole is sufficiently strong.

Figure 8 is the phase diagram for $\mu^{*}=2.5$. A strongly first-order phase transition, directly from the isotropic fluid to the smectic-B phase, is observed. The nature of the transition is made clear by a jump in the bond orientational order at the I-LC transition. Alignment of the molecules is accompanied not only by layering, but also the development of long-ranged bond order within these layers. The bond-order parameter takes a value of $B=0.55$ at the transition. For these highly polar GB molecules, the I-SmB transition oc- 

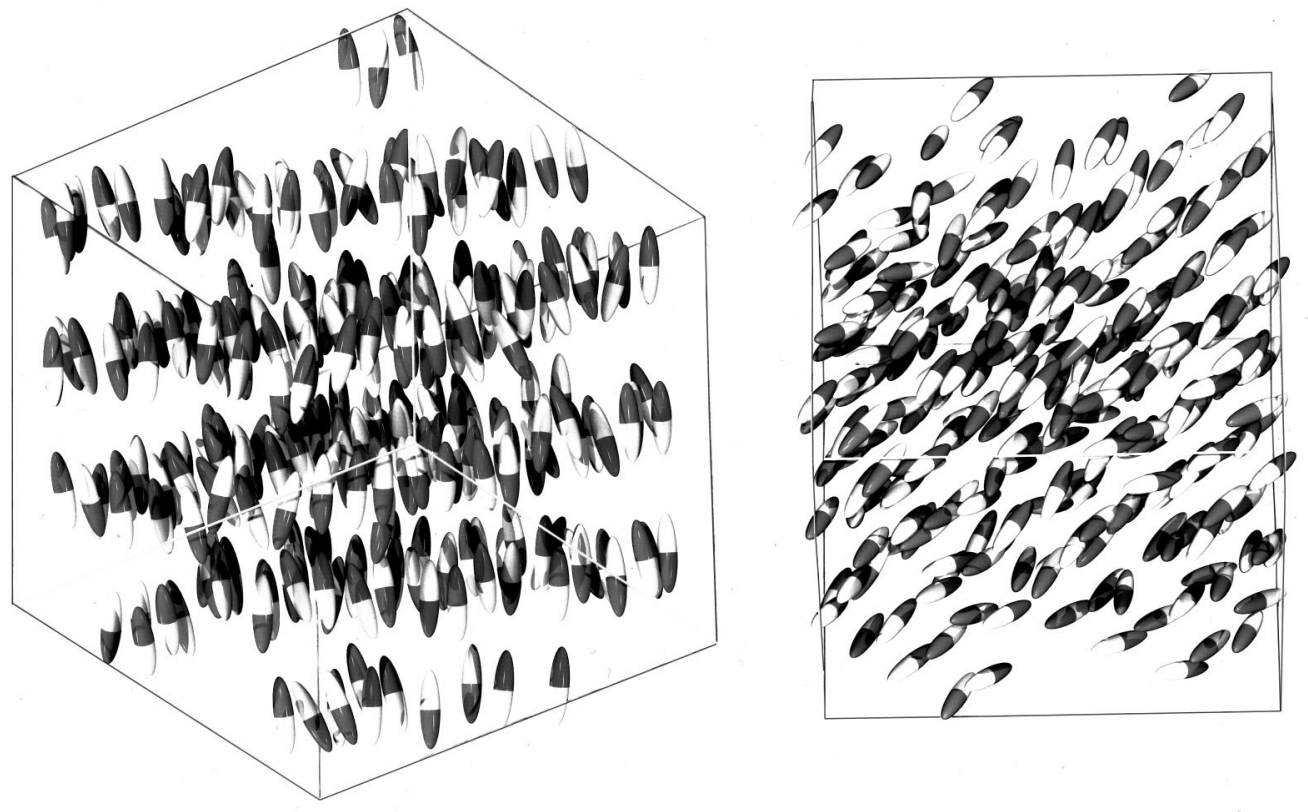

FIG. 5. Snapshot of the dipolar Gay-Berne fluid at $T^{*}=1.25, P^{*}=9.0, \mu^{*}=1.5$, in the smectic-B phase. The same configuration is shown from different perspectives: (a) from the side to show the smectic layering and (b) exhibiting the strong positional correlation between successive layers. The colors indicate the direction of the dipole. The size of the particles is reduced for clarity.
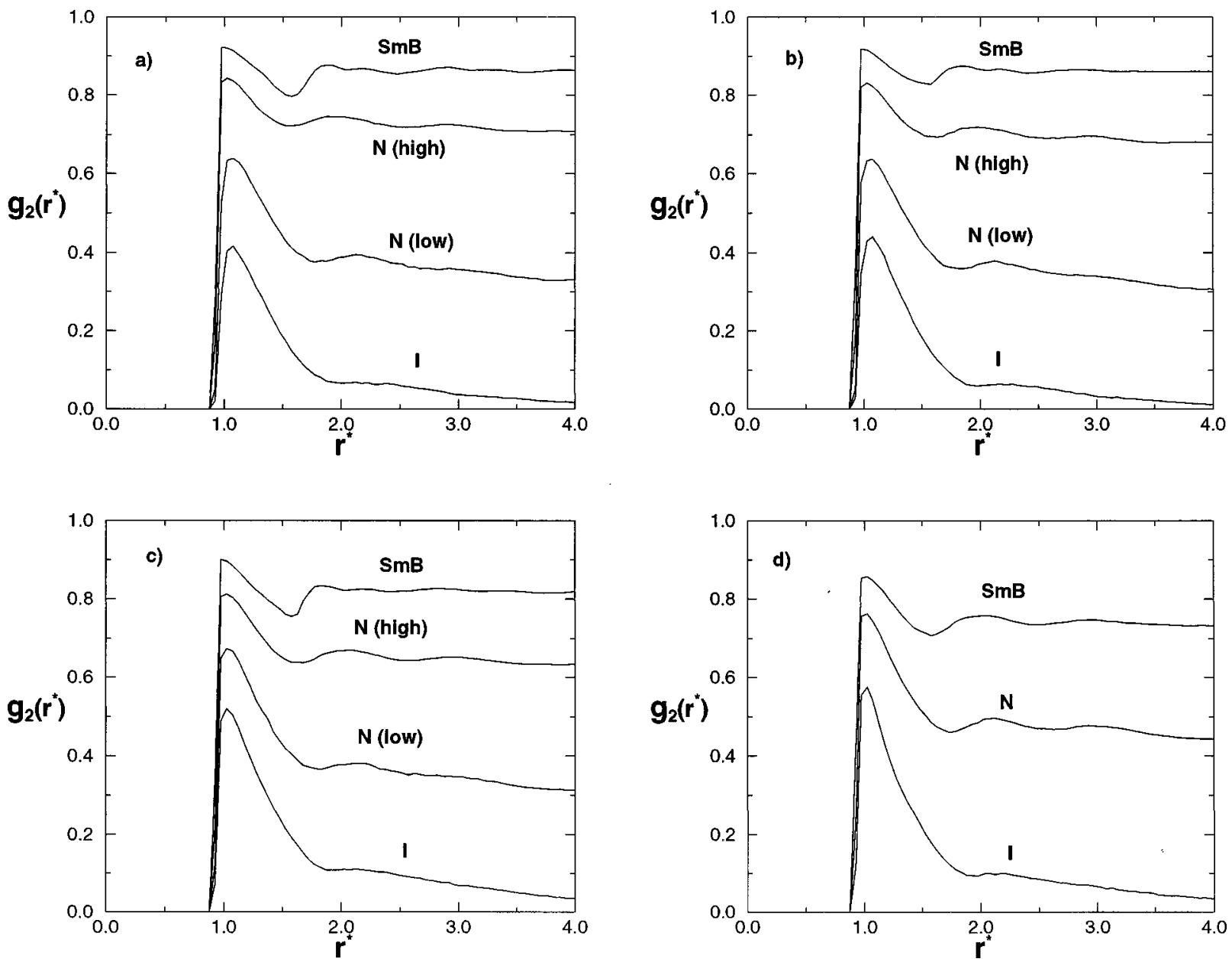

FIG. 6. Second-rank orientational correlation function $g_{2}(r)$ for dipolar Gay-Berne fluids at the I-N and N-SmB transitions. The four figures represent different dipole moments (as for Fig. 1). 

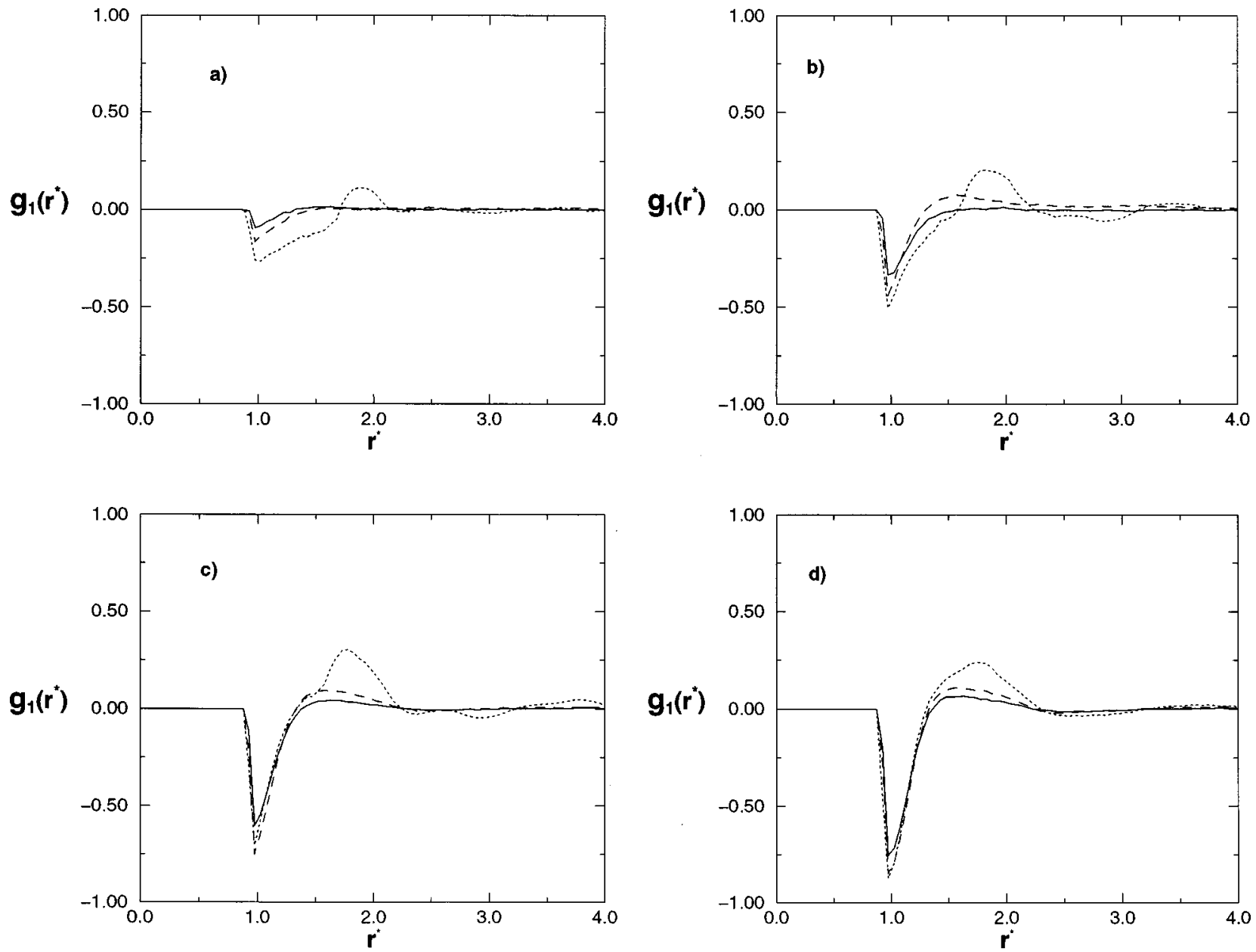

FIG. 7. First-rank orientational correlation function $g_{1}(r)$ for dipolar Gay-Berne fluids in the isotropic, nematic, and smectic-B phases. The four figures represent different dipole moments (as for Fig. 1).

curs at a significantly lower pressure than the I-N transition for the weaker dipolar systems. Once again, though, we note from Table I that the density at which the isotropic phase ceases to be stable is unaffected $\rho^{*} \approx 0.305$.

The position of the phase transition is inferred from the pressure versus density phase diagram, Fig. 8. Even more compelling evidence for the location of the phase transition is provided by the behavior of the nematic order parameter $S$

TABLE I. Transition pressures and densities for dipolar GB fluid at a reduced temperature of $T^{*}=1.25$ as obtained by MCNPT simulation. GB parameters are: $\kappa=3, \kappa^{\prime}=5 . \mu^{*}$ is the reduced dipole moment, $P_{\mathrm{IN}}$ is the isotropic to nematic transition pressure, $P_{\mathrm{NSmB}}$ is the same property for the nematic to smectic-B transition, $\rho_{\mathrm{IN}}$ are the densities of the coexisting isotropic and nematic phases, and $\rho_{\mathrm{NSmB}}$ are the same values for the layering transition. Note that the strongest dipole has a direct isotropic to smectic-B transition. The system size is $N=256$.

\begin{tabular}{ccccc}
\hline \hline$\mu^{*}$ & $P_{\mathrm{IN}}$ & $\rho_{\mathrm{IN}}$ & $P_{\mathrm{NSmB}}$ & $\rho_{\mathrm{NSmB}}$ \\
\hline 0.5 & 4.99 & $0.304,0.319$ & 9.49 & $0.368,0.380$ \\
1.0 & 5.00 & $0.305,0.320$ & 9.00 & $0.364,0.379$ \\
1.5 & 5.00 & $0.307,0.322$ & 7.00 & $0.346,0.361$ \\
2.0 & 4.49 & $0.305,0.321$ & 4.99 & $0.321,0.339$ \\
& $P_{\mathrm{ISmB}}$ & $\rho_{\mathrm{ISmB}}$ & & \\
2.5 & 3.70 & $0.300,0.348$ & & \\
\hline \hline
\end{tabular}

and polarity $P_{1}$ as a function of density (Fig. 9). The variation of the order parameters also provides information as to the nature of the resultant phases. The first nonzero value of $S$ occurs at $\rho^{*} \approx 0.348$, but now we can see notable pretran-

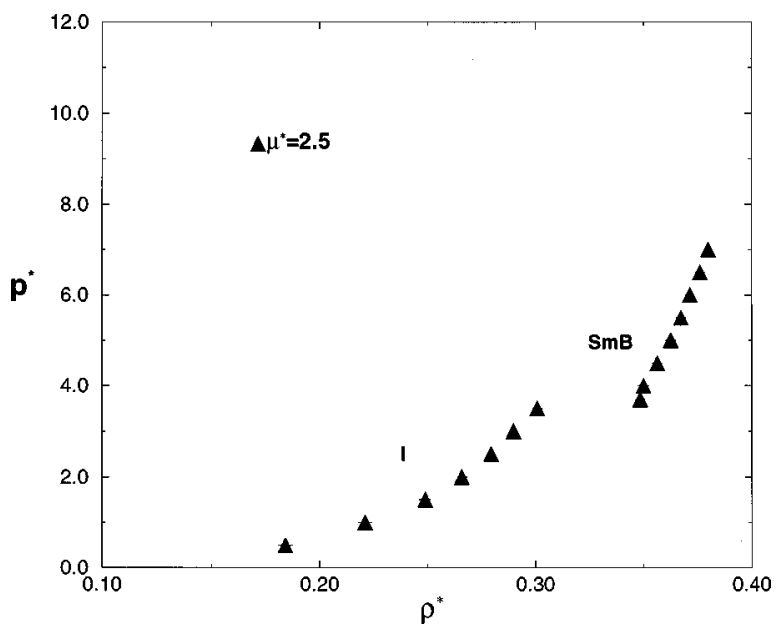

FIG. 8. Phase diagram at $T^{*}=1.25$, of a system of 256 Gay-Berne particles $\left(\kappa=3, \kappa^{\prime}=5\right)$ with embedded central, longitudinal point dipoles. The reduced pressure $P^{*}=P \sigma_{0}^{3} / \varepsilon_{0}$ is plotted as a function of the reduced number density $\rho^{*}=\rho \sigma_{0}^{3}$. The reduced dipole moment is $\mu^{*}=\left(\mu^{2} / \varepsilon_{0} \sigma_{0}^{3}\right)^{1 / 2}=2.5$. Error bars represent one standard error in the density. 


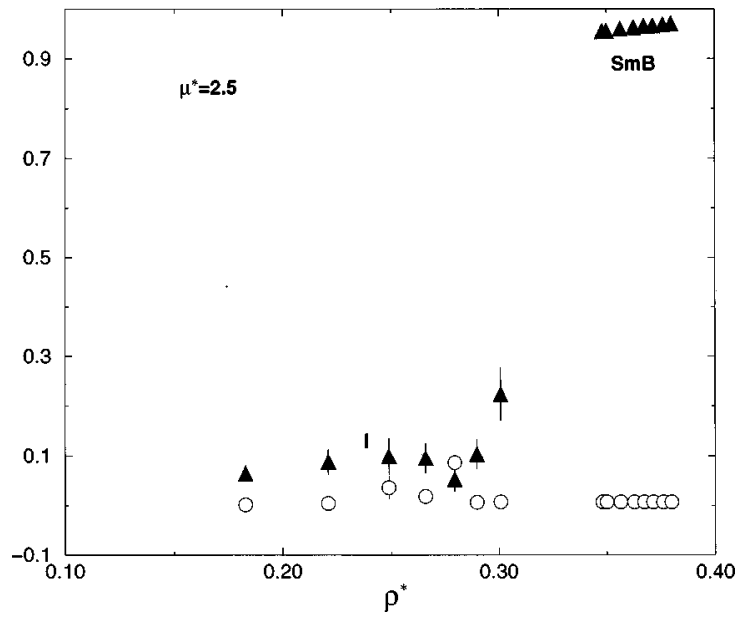

FIG. 9. Variation of the nematic order parameter $S$ (triangles) and polarity $P_{1}$ (circles), as a function of density for the dipolar Gay-Berne fluid. The reduced dipole moment is $\mu^{*}=2.5$. Error bars denote one standard error in the order parameters.

sitional fluctuations (a feature which was absent for the weaker dipolar systems). This transition is directly to very high values $S \approx 0.85$, consistent with a smectic liquid crystal. Figure 10 is the projection of the radial pair distribution function along the director on both sides of this transition. The sinusoidal variation of this function confirms that the liquid crystal phase is a smectic. As mentioned above, the bond-orientational order becomes nonzero at the same pressure. We thus conclude that the transition is directly from the isotropic to the smectic-B phase.

It should be pointed out that the exact location of the type of triple point $(\mathrm{I}-\mathrm{N}-\mathrm{SmB})$ suggested by our results, by simulation is extremely difficult, since three phases coexist. Our best estimate is that for this model at this temperature $\left(T^{*}=1.25\right)$, the $\mathrm{I}-\mathrm{N}-\mathrm{SmB}$ triple point is very close to $\mu^{*}$ $=2.0$.

From Fig. 9 we again reiterate many previous findings: such systems show absolutely no evidence of spontaneous polar order. The function $P_{1}$ shows some fluctuations around

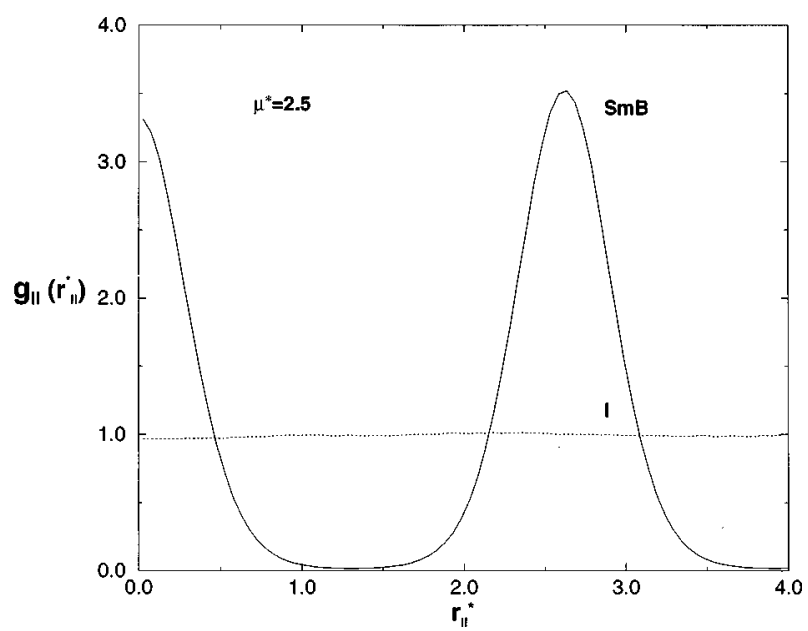

FIG. 10. The projection of the radial pair distribution function parallel to the director $g_{\|}\left(r_{\|}\right)$for states on either side of the isotropic-smectic transition for the dipolar Gay-Berne fluid. $\kappa=3, \kappa^{\prime}=5, T^{*}=1.25$, and $\mu^{*}=2.5$.

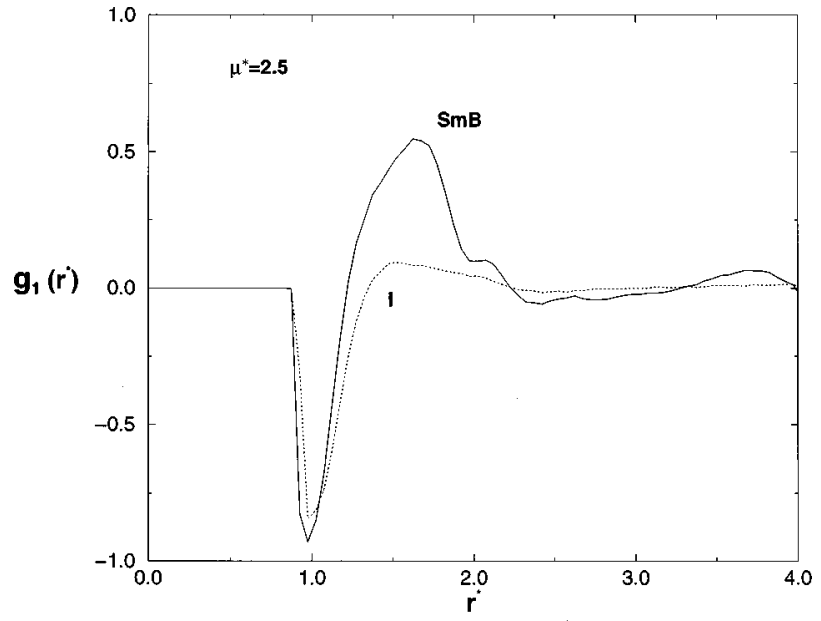

FIG. 11. The first-rank orientational correlation function $g_{1}(r)$ as a function of separation, for the dipolar Gay-Berne fluid, on either side of the I-Sm transition. $\kappa=3, \kappa^{\prime}=5, T^{*}=1.25$, and $\mu^{*}=2.5$.

the phase transitions, but all the values are consistent with nonpolar phases. Polar phases are most likely in the highly ordered smectic-B phase of the strongly polar system; we show in Fig. 11 the variation of the first-rank orientational correlation function $g_{1}(r)$. As expected, the dipole induces antiparallel order in nearest neighbors, and parallel order in the next coordination shell, but this function is not long ranged. We confidently repudiate polar phases for this system.

As for the weaker dipolar models, we have performed simulations from high pressure to monitor the hysteresis associated with the phase transitions. For these stronger dipoles, we note a significant degree of hysteresis, particularly in the pressure.

\section{High temperature, $\mu^{*}=2.5$}

For the same GB model, we have investigated the influence of temperature on the phase diagram for fixed dipole

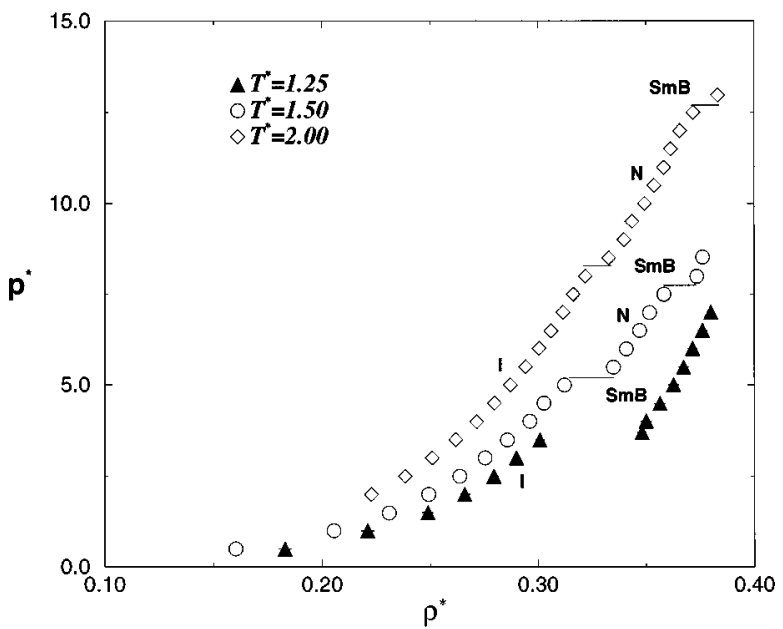

FIG. 12. Phase diagrams for a system of 256 Gay-Berne particles $(\kappa=3$, $\left.\kappa^{\prime}=5\right)$ with embedded central, longitudinal point dipoles with dipole moment $\mu^{*}=\left(\mu^{2} / \varepsilon_{0} \sigma_{0}^{3}\right)^{1 / 2}=2.5$. Triangles are $T^{*}=1.25$, circles $T^{*}=1.5$, and diamonds $T^{*}=2.0$. Error bars represent one standard error in the density. 
TABLE II. Transition pressures and densities for dipolar GB fluid at a reduced dipole moment of $\mu^{*}=2.5$ as obtained by MCNPT simulation. GB parameters are: $\kappa=3, \kappa^{\prime}=5$. The reduced temperature is $T^{*}, P_{\mathrm{IN}}$ is the isotropic to nematic transition pressure, $P_{\mathrm{NSmB}}$ is the same property for the nematic to smectic-B transition, $\rho_{\text {IN }}$ are the densities of the coexisting isotropic and nematic phases, and $\rho_{\mathrm{NSmB}}$ are the same values for the layering transition. Note that the coldest system exhibits a direct isotropic to smectic-B transition. The system size is $N=256$.

\begin{tabular}{cccrc}
\hline \hline$T^{*}$ & $P_{\mathrm{IN}}$ & $\rho_{\mathrm{IN}}$ & $P_{\mathrm{NSmB}}$ & $\rho_{\mathrm{NSmB}}$ \\
\hline 2.00 & 8.50 & $0.332,0.339$ & 12.50 & $0.371,0.383$ \\
1.50 & 5.49 & $0.312,0.334$ & 7.99 & $0.358,0.373$ \\
& $P_{\mathrm{ISmB}}$ & $\rho_{\mathrm{ISmB}}$ & & \\
1.25 & 3.70 & $0.300,0.348$ & & \\
\hline \hline
\end{tabular}

moment. Several isotherms have been constructed for a reduced dipole moment of $\mu^{*}=2.5$. This strong dipole yields only an isotropic and smectic-B phase at the temperature discussed above $\left(T^{*}=1.25\right)$. Compression isotherms at two higher temperatures, $T^{*}=1.5$ and 2.0, are depicted in Fig. 12 and the transitions are detailed in Table II.

At $T^{*}=1.5$ a nematic phase is observed between the isotropic and smectic-B phases. The range of densities over which this phase is stable is seen to increase at the higher temperature $T^{*}=2.0$.

The transition pressures are clearly very sensitive to the temperature. The pressure at which the isotropic phase becomes unstable decreases as the temperature is lowered. The transition density is again less sensitive to the changes. The maximum density at which the isotropic phase may be observed increases slightly with increasing temperature, but at the same time, the biphasic region narrows. Thus little can be inferred from this observation.

\section{B. $\kappa=4$}

Systems with a larger value of $\kappa$, i.e., more elongated molecules, are known to have a wider range of stability of the liquid crystal phases. The density at which the I-N transition occurs is lower for $\kappa=4\left(\rho_{I} \approx 0.19, \rho_{\mathrm{N}} \approx 0.20\right)^{28}$ than for $\kappa=3\left(\rho_{I} \approx 0.315, \rho_{\mathrm{N}} \approx 0.32\right),{ }^{29}$ at $T^{*}=1.25$. The stability of the smectic phase is similarly affected: for $\kappa=4, \rho_{\mathrm{N}}$ $\approx 0.21, \rho_{\mathrm{Sm}} \approx 0.22,{ }^{28}$ whereas for $\kappa=3, \rho_{\mathrm{N}} \approx 0.37, \rho_{\mathrm{Sm}}$ $\approx 0.375 .{ }^{29}$

From the work of Brown ${ }^{30}$ we know that for $\kappa=4$, at the temperature $T^{*}=1.25$, the low density isotropic phase is separated from the high density smectic-B phase by not only a nematic phase (as is the case for $\kappa=3$ ) but also by a smectic-A phase. Allen et al. ${ }^{31}$ suggest that the smectic-A phase is stable only for $\kappa \geqslant 3.4$. The phase diagram of Brown et al. ${ }^{28}$ is very interesting, with the nematic region ending at higher temperatures than the smectic-A phase, i.e., at an I$\mathrm{N}-\mathrm{SmA}$ triple point. At lower temperatures, compression of the isotropic fluid leads directly to a smectic-A phase. The region of stability of the smectic-A phase is also bounded at low temperatures by a triple point (I-SmA-SmB), but very interestingly the phase also becomes unstable at high temperatures. Consequently, at temperatures above this $\mathrm{N}-$ SmA-SmB triple point, the nematic phase transforms di-

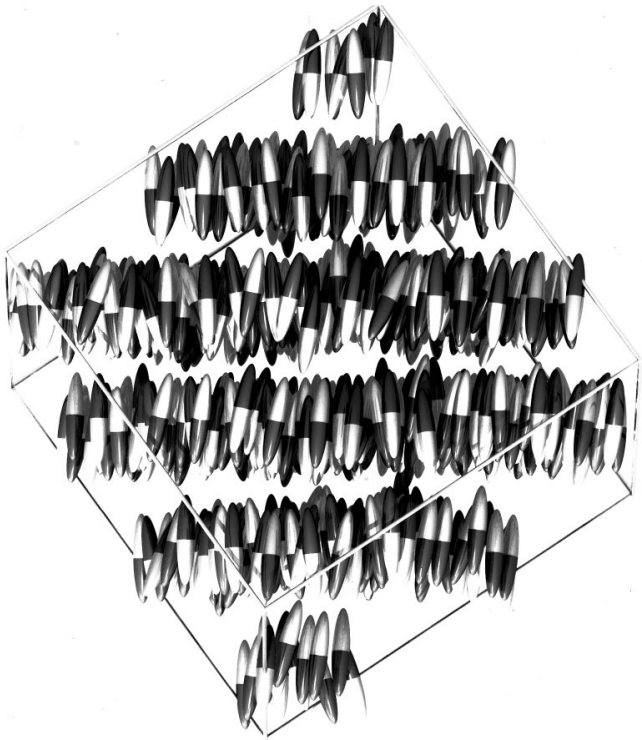

FIG. 13. Snapshot of a system of $500 \mathrm{~GB}$ molecules $\left(\kappa=4, \kappa^{\prime}=5\right)$ with central longitudinal point dipoles. In this case $\mu^{*}=2.0, P^{*}=2.5$. The colors indicate the orientation of the dipole. The size of the particles is reduced for clarity.

rectly to the smectic-B phase upon compression. This "island" of smectic-A stability is an unusual feature.

We have performed isothermal-isobaric simulations at $T^{*}=1.25$ for a system of $N=500$ particles in a cuboidal simulation cell, at three pressures $P^{*}=1.5,2.0$, and 2.5. At this temperature, in the absence of dipolar forces these pressures correspond to isotropic, nematic, and smectic-A phases, respectively. The addition of longitudinal point dipoles at the center of the molecule is seen to have very little effect on the thermodynamic state of the system, for all dipole moments $\mu^{*} \leqslant 1.5$ (see Table III). For such dipole strengths, the density and internal energy both increase slightly with dipole moment, as does the nematic order parameter in the liquid crystalline phases. No globally polar order is noted, and the structure of the phases remains unaffected by the presence of these weak multipoles.

Far more drastic effects are noted for stronger dipoles. With $\mu^{*}=2.0$, the lowest pressure state is a smectic-A phase. The system evolves to a smectic-B phase by the highest pressure studied $\left(P^{*}=2.5\right)$, see Fig. 13. From this snapshot, the layering is seen to be very distinct, with few particles diffusing between layers. The highly ordered nature of this phase is confirmed by inspection of the various pair distribution functions in Fig. 14. The first-rank orientational correlation function [Fig. 14(a)] has more structure than previously observed. The short-range antiferroelectric order is now seen to persist over at least four coordination shells. The second-rank orientational correlation function [Fig. 14(b)] indicates the very high orientational order present in the system. The projections of the radial pair distribution functions along [Fig. 14(c)] and perpendicular [Fig. 14(d)] to the director show that the phase has strong positional correlations, indicative of a highly ordered smectic-B phase. It should be noted that Allen et al. ${ }^{31}$ argued that there is no distinction 
TABLE III. MCNPT results obtained for GB dipolar fluids with $\kappa=4, \kappa^{\prime}=5$ at $T^{*}=1.25, \mu^{*}$ is the reduced dipole moment, $\rho^{*}$ the reduced density, $P^{*}$ the corresponding reduced pressure, $B$ the bond orientational order parameter, $S$ the orientational order parameter, and $P_{1}$ is the first-order parameter. The system size is $N$ $=500$.

\begin{tabular}{ccccccc}
\hline \hline$\mu^{*}$ & $\rho^{*}$ & $U^{*}$ & \multicolumn{1}{c}{$S$} & $P_{1}$ & $B$ & Phase \\
\hline & & & $P^{*}=1.5$ & & \\
0.0 & $0.17959(106)$ & $-2.53589(3472)$ & $0.21098(1999)$ & & $0.005(11)$ & $I$ \\
0.5 & $0.17879(144)$ & $-2.40939(4313)$ & $0.13887(4777)$ & $-0.01446(2677)$ & $0.007(10)$ & $I$ \\
1.0 & $0.17899(140)$ & $-2.50317(4394)$ & $0.11116(2577)$ & $-0.00182(2390)$ & $0.008(10)$ & $I$ \\
1.5 & $0.18128(128)$ & $-2.89671(5596)$ & $0.12536(3607)$ & $0.00341(2389)$ & $0.006(11)$ & $I$ \\
2.0 & $0.20969(171)$ & $-5.71070(14786)$ & $0.87824(1041)$ & $-0.01629(301)$ & $0.017(33)$ & Sm A \\
2.5 & $0.22276(146)$ & $-8.66233(10273)$ & $0.93089(484)$ & $-0.00778(98)$ & $0.028(54)$ & Sm A \\
& & & $P^{*}=2.0$ & & & \\
0.0 & $0.20766(141)$ & $-3.21932(5801)$ & $0.75436(1950)$ & & $0.026(11)$ & $\mathrm{N}$ \\
0.5 & $0.20907(127)$ & $-3.27047(5783)$ & $0.76704(1630)$ & $0.00294(397)$ & $0.005(15)$ & $\mathrm{N}$ \\
1.0 & $0.20997(166)$ & $-3.49479(11611)$ & $0.77716(2049)$ & $0.01637(653)$ & $0.002(15)$ & $\mathrm{N}$ \\
1.5 & $0.21078(143)$ & $-4.07378(9640)$ & $0.81547(1531)$ & $-0.01243(364)$ & $0.001(22)$ & $\mathrm{N}$ \\
2.0 & $0.22684(155)$ & $-6.72465(14783)$ & $0.91918(750)$ & $0.00092(70)$ & $0.025(44)$ & Sm A \\
2.5 & $0.23945(149)$ & $-9.05840(8815)$ & $0.95024(326)$ & $-0.03133(70)$ & $0.447(51)$ & Sm B \\
& & & & & \\
0.0 & $0.22615(141)$ & $-4.02357(12319)$ & $0.87736(1272)$ & & $0.072(23)$ & Sm A \\
0.5 & $0.22522(122)$ & $-4.01623(10572)$ & $0.86704(1027)$ & $-0.01181(175)$ & $0.007(30)$ & Sm A \\
1.0 & $0.22646(121)$ & $-4.04228(9378)$ & $0.88222(937)$ & $-0.00106(244)$ & $0.012(33)$ & Sm A \\
1.5 & $0.23048(131)$ & $-5.02140(8804)$ & $0.90670(715)$ & $-0.00383(136)$ & $0.018(57)$ & Sm A \\
2.0 & $0.24364(194)$ & $-7.40875(13321)$ & $0.94733(617)$ & $0.00059(44)$ & $0.559(53)$ & Sm B \\
2.5 & $0.24904(115)$ & $-9.25047(7155)$ & $0.95865(259)$ & $-0.00393(56)$ & $0.318(94)$ & Sm B \\
\hline \hline & & & & & &
\end{tabular}

between the smectic-B and crystalline phases for the nonpolar $\kappa \geqslant 3$ GB model.

A similar phase sequence is noted for $\mu^{*}=2.5$, but the smectic-B phase is now stable for pressures as low as $P^{*}$ $=2.0$. Hence, the smectic phases are strongly stabilized by the dipole for this value of $\kappa$. It is of interest to know if the stabilization of the smectic phases is at the expense of the nematic or isotropic phase. For spherocylinders with a similar I-N-SmA phase sequence, the incorporation of strong dipole moments led to a complete suppression of the nematic phase, and a direct I-SmA transition. ${ }^{14}$ Is the same true of GB systems?

We explored the low pressure region of the phase diagram for these two dipole strengths. For $\mu^{*}=2$, stepping down in pressure, the orientational and positional order are both seen to terminate at a pressure $P^{*}=0.75$. This would appear to indicate that the nematic phase does not exist for this combination of elongation, temperature, and dipole moment. However, the quite large hysteresis that can be observed at the LC transitions often masks thermodynamically stable states. In order to investigate this possibility, we commence simulations from nematic phases at $P^{*}=1.0$ and
1.25. In both cases the system evolves to an isotropic phase. Thus, we conclude that there is no stable nematic phase for this system at this temperature. Of course at higher temperatures, where the influence of the dipole diminishes, the nematic phase may again become stable.

We perform a sequence of simulations for the strongest dipole $\mu^{*}=2.5$, at an intermediate pressure $P^{*}=1.5$, varying the temperature. As noted above, these parameters correspond to a smectic-A phase at $T^{*}=1.25$. Cooling the system $\left(T^{*}=1.0\right)$ results in the evolution of in-plane bond order, and the phase may be identified as smectic-B. Warming the system increases the symmetry of the phase, with the positional order being lost by a temperature of $T^{*}=1.5$, and the orientational order coming to an end at a higher temperature $T^{*}=1.75$. The phase sequence for this elongation and dipole strength can be identified as $\mathrm{I}-\mathrm{N}-\mathrm{SmA}-\mathrm{SmB}$ as the temperature is lowered (see Table IV).

These results are in accordance with expectation. The strong affinity for dipolar molecules promotes layered phases. Stabilization of the smectic phases is noted. For sufficiently strong dipoles, the nematic phase can be preempted by an I-Sm transition. However, raising the temperature sta-

TABLE IV. MCNPT results obtained for dipolar GB fluids with $\kappa=4, \kappa^{\prime}=5$ at reduced pressure $P^{*}=1.5 . T^{*}$ is the reduced temperature and $\rho^{*}$ the reduced density. The value of the reduced dipole moment is $\mu^{*}=2.5, B$ the bond orientational order parameter, $S$ the orientational order parameter, and $P_{1}$ is the first-order parameter. The system size is $N=500$.

\begin{tabular}{|c|c|c|c|c|c|c|}
\hline$T^{*}$ & $\rho^{*}$ & $U^{*}$ & $S$ & $P_{1}$ & $B$ & Phase \\
\hline \multicolumn{7}{|c|}{$P^{*}=1.5$} \\
\hline 1.00 & $0.24364(147)$ & $-9.98756(6312)$ & $0 . \overline{96299(264)}$ & $-0.00795(51)$ & $0.452(50)$ & Sm B \\
\hline 1.25 & $0.22276(146)$ & $-8.66233(10273)$ & $0.93089(484)$ & $-0.00778(98)$ & $0.028(54)$ & $\mathrm{Sm} \mathrm{A}$ \\
\hline 1.50 & $0.20013(259)$ & $-6.60458(24526)$ & $0.84367(2462)$ & $-0.01090(476)$ & $0.013(27)$ & $\mathrm{N}$ \\
\hline 1.75 & $0.16356(129)$ & $-3.58256(8168)$ & $0.09024(2865)$ & $-0.00455(1711)$ & $0.007(10)$ & I \\
\hline
\end{tabular}



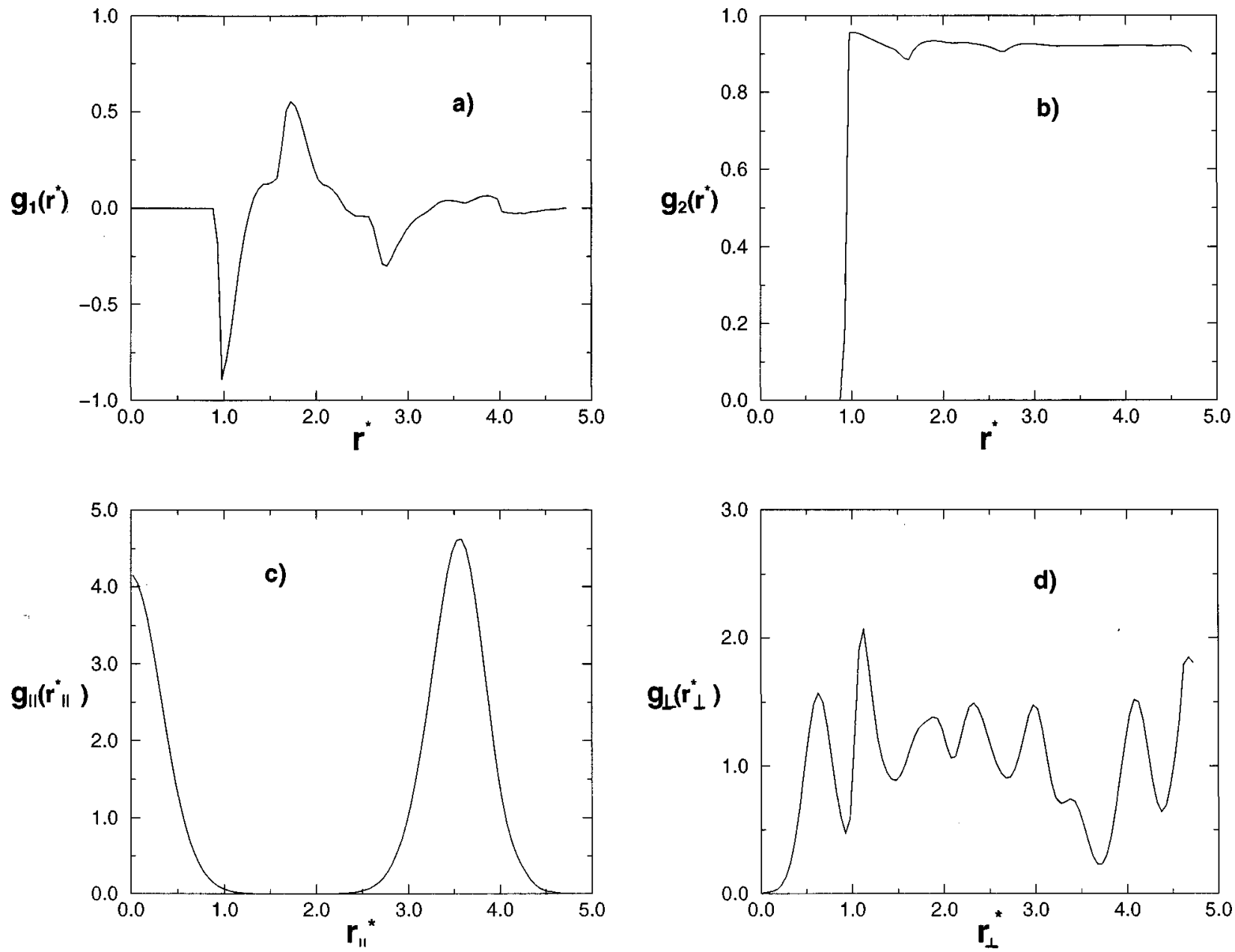

FIG. 14. Distribution functions for the dipolar GB in the smectic-B phase. The same state point as Fig. 13 is depicted. (a) The first-rank orientational correlation function $g_{1}(r)$ as a function of separation; (b) second-rank orientational correlation function $g_{2}(r)$ as a function of separation; (c) projection of the radial pair distribution function parallel to the director $g_{\|}\left(r_{\|}\right)$; (d) projection of the radial pair distribution function perpendicular to the director $g_{\perp}\left(r_{\perp}\right)$.

bilizes the positionally disordered LC phase once more. Globally, therefore, increasing the dipole moment elevates the I-N-Sm triple point. Note that we do not definitively say which smectic phase the isotropic and nematic phases coexist with, although presumably since for nonpolar $\kappa=4 \mathrm{~GB}$ systems the smectic-A phase is stable at lower temperatures than is the nematic phase, it will be the SmA phase that will coexist with the isotropic phase just below this triple point.

\section{CONCLUSIONS}

The rich and well-documented phase behavior of the Gay-Berne fluid makes a system of such molecules an ideal candidate for use in the study of the effect of perturbations (such as dipolar forces) on the observed phase diagram.

As in many previous studies, we note the complete absence of polar phases. Around the phase transitions, some fluctuations in $P_{1}$ can be seen, but these do not persist. Phases with $P_{1}=0$ may still be antiferroelectric, but the first-rank orientational correlation function remains short ranged for all the simulations performed here, hence this type of polar order is also absent. The most significant impact that central longitudinal dipoles have is the increased stability of the layered smectic phases. As the strength of the dipole is increased, the density of the transition to the smectic phase (from either the isotropic or the nematic phase) decreases. For a GB model which displays both nematic and smectic phases without multipolar forces, the nematic phase eventually becomes unstable and the system aligns directly from the isotropic to the smectic phase. Thus a kind of triple point may be envisaged: at a certain dipolar strength all three phases can coexist.

A remarkable feature of all the simulations is the insensitivity of the initial I-LC transition to the strength of the multipole. For a given temperature, the maximum density of the isotropic phase varies by less than $1 \%$ as the reduced dipole moment varies from $\mu^{*}=0$ to 2.5 . The effect on the transition pressure is larger, but only becomes significant when the dipole is strong enough to influence the nature of the resultant mesophase. For strong dipoles, a direct I-SmB transition is noted $(\kappa=3)$ and the transition pressure is much lower than the I-N transition pressure associated with the more weakly dipolar systems. This constancy of the I-LC transition density is different from the behavior noted for hard-core mesogens such as the spherocylinder. For such systems, the inclusion of the dipole postpones the transition significantly (although hysteresis at the transition means that 
the LC phase persists down at least as far as the original transition density). This qualitative difference presumably stems from the fact that there is a strong side-by-side attraction already present in the GB system, prior to incorporation of the dipole. In purely repulsive systems, the inclusion of dipoles creates totally new interactions, whereas in the GB system the dipole reinforces the already existing attractions.

A noteworthy result is the stabilization of the highly ordered smectic-B phase, at the expense of the smectic-A liquid crystal. The two phases are distinguishable due to the long-ranged, in-plane bond order which is present in the former, but absent in the latter. This, of course, begs the question, why should this bond order be enhanced by the presence of a central dipole? Careful analysis of the results indicates that for $\kappa=4$, the density at which the SmA-SmB transition occurs has very little $\mu^{*}$ dependence. For the system with $\kappa=3$, there is no smectic-A phase; when the system layers, the bond order also becomes long ranged. Now there is a very real difference in structure depending on the strength of the multipole. For $\mu^{*}=0.5$, the nematic phase is stable to a density of $\rho^{*}=0.37$, but at $\mu^{*}=2.0$, the smectic-B phase is already stable at densities as low as $\rho^{*}$ $=0.34$. Our interpretation of these results is that the strong dipole is favoring the layered structure (i.e., smectic phases in general), but the shape of the ellipsoidal core is primary in stabilizing the smectic-B phase. Thus although for $\kappa=4$ the SmA-SmB transition can be brought about in an NPT simulation, simply by increasing the strength of the dipole, this phase change occurs entirely because the density of the system increases with dipole moment. The same arguments hold for the system of shorter ellipsoids ( $\kappa=3$ ), but now we contend that the phase diagram is such that all densities which correspond to layered structures are higher than the minimum density required to bring about long-range bond order. Hence, the $\mathrm{N}-\mathrm{SmB}$ transition can be brought about by increasing the dipole moment, since dipoles promote layered structures. The bond order exists because the density of the resultant phase is beyond the threshold of smectic-A stability. It is not inconceivable that stronger dipoles might create a layered structure at densities sufficiently low to allow smectic-A phases to be seen for this elongation and temperature. However, it may be that the density at which the smectic-A phase would be stable is lower than the minimum density required for orientational order, and since we note that the maximum density of the isotropic phase is impervious to the dipole, a bulk smectic-A phase might very well be precluded.

We note from the various distribution functions that the degree of interdigitation in the smectic phases is high, but insensitive to the dipolar forces. Presumably the layer spacing of less than one molecular length stems from the shape of the molecular cores. The utility of accumulating several distribution functions has been clearly demonstrated. In order to unambiguously determine the structure of the system, information from a variety of pair correlation functions, order parameters, and direct visualization of the configuration must be combined.
Additionally, the efficacy of the reaction field method has once again been demonstrated. This technique is many times faster than the Ewald summation method, and thus allows much greater regions of the phase diagram to be probed. Recent studies ${ }^{9,24,25}$ have conclusively shown that, provided system sizes are adequate, the reaction field method is no less accurate than the Ewald scheme.

\section{ACKNOWLEDGMENTS}

S.C.M. thanks the National Science Foundation for a CISE Post-Doctoral Fellowship. Financial support from the Dirección General de Investigación Científica y Técnica (Spain), PB94-1442, Plan Andaluz de Investigación and European Union (ERB4050 PL 930367 and CI1*-CT94-0132) are gratefully acknowledged.

${ }^{1}$ D. Frenkel, J. Phys. Chem. 92, 3280 (1988).

${ }^{2}$ A. Stroobants, H. N. W. Lekkerkerker, and D. Frenkel, Phys. Rev. A 36, 2929 (1987).

${ }^{3}$ D. Photinos and E. Samulski, Science 270, 783 (1995).

${ }^{4}$ D. Frenkel and B. M. Mulder, Mol. Phys. 55, 1171 (1985).

${ }^{5}$ J. A. C. Veerman and D. Frenkel, Phys. Rev. A 41, 3237 (1990).

${ }^{6}$ L. Onsager, Phys. Rev. 62, 558 (1942).

${ }^{7}$ L. Onsager, Ann. (N.Y.) Acad. Sci. 51, 627 (1949).

${ }^{8}$ E. de Miguel, L. F. Rull, M. K. Chalam, K. E. Gubbins, and F. van Swol, Mol. Phys. 72, 593 (1991).

${ }^{9}$ M. Houssa, A. Oualid, and L. F. Rull, Mol. Phys. 94, 439 (1998).

${ }^{10}$ D. Levesque, J. J. Weis, and G. J. Zarragoicoechea, Phys. Rev. E 47, 496 (1993).

${ }^{11}$ J. J. Weis, D. Levesque, and G. J. Zarragoicoechea, Phys. Rev. A 46, 7783 (1992).

${ }^{12}$ G. J. Zarragoicoechea, D. Levesque, and J. J. Weis, Mol. Phys. 75, 989 (1992); 74, 629 (1991).

${ }^{13}$ G. J. Zarragoicoechea, D. Levesque, and J. J. Weis, Mol. Phys. 78, 1475 (1993).

${ }^{14}$ S. C. McGrother, A. Gil-Villegas, and G. Jackson (in preparation).

${ }^{15}$ A. Gil-Villegas, S. C. McGrother, and G. Jackson, Chem. Phys. Lett. 269, 441 (1997).

${ }^{16}$ S. C. McGrother, A. Gil-Villegas, and G. Jackson, J. Phys.: Condens. Matter 8, 9649 (1996).

${ }^{17}$ K. Satoh, S. Mita, and S. Kondo, Liq. Cryst. 20, 757 (1996).

${ }^{18}$ K. Satoh, S. Mita, and S. Kondo, Chem. Phys. Lett. 255, 99 (1996).

${ }^{19}$ R. Berardi, S. Orlandi, and C. Zannoni, Chem. Phys. Lett. 261, 357 (1996).

${ }^{20}$ E. Gwóźdź, A. Bródka, and K. Pasterny, Chem. Phys. Lett. 267, 557 (1997).

${ }^{21}$ J. G. Gay and B. J. Berne, J. Chem. Phys. 74, 3316 (1981).

${ }^{22}$ J. A. Barker and R. O. Watts, Mol. Phys. 26, 789 (1973).

${ }^{23}$ M. Neumann, Mol. Phys. 50, 841 (1983).

${ }^{24}$ A. Gil-Villegas, S. C. McGrother, and G. Jackson, Mol. Phys. 92, 723 (1997).

${ }^{25}$ B. Garzon, S. Lago, and C. Vega, Chem. Phys. Lett. 231, 366 (1994).

${ }^{26}$ C. Zannoni, The Molecular Physics of Liquid Crystals, edited by G. R. Luckhurst and G. W. Gray (Academic, New York, 1979).

${ }^{27}$ J. S. vanDuijneveldt and M. P. Allen, Mol. Phys. 90, 243 (1997).

${ }^{28}$ J. T. Brown, M. P. Allen, E. M. del Rio, and E. de Miguel, Phys. Rev. E 57, 6685 (1998).

${ }^{29}$ E. de Miguel, L. F. Rull, M. K. Chalam, and K. E. Gubbins, Mol. Phys. 74, 405 (1991).

${ }^{30}$ J. T. Brown, Ph.D. thesis, University of Bristol, 1997.

${ }^{31}$ M. P. Allen, J. T. Brown, and M. A. Warren, J. Phys.: Condens. Matter 8, 9433 (1996) 